REVIEW ARTICLE

\title{
Challenges and translational considerations of mesenchymal stem/stromal cell therapy for Parkinson's disease
}

\author{
Dominika Fričová ${ }^{1,2}$, Jennifer A. Korchak ${ }^{1}$ and Abba C. Zubair $\mathbb{I D}^{1 凶}$
}

Parkinson's disease (PD) is the second most common neurodegenerative disease characterized by the progressive loss of dopaminergic neurons in the substantia nigra pars compacta and the presence of Lewy bodies, which gives rise to motor and nonmotor symptoms. Unfortunately, current therapeutic strategies for PD merely treat the symptoms of the disease, only temporarily improve the patients' quality of life, and are not sufficient for completely alleviating the symptoms. Therefore, cell-based therapies have emerged as a novel promising therapeutic approach in PD treatment. Mesenchymal stem/stromal cells (MSCs) have arisen as a leading contender for cell sources due to their regenerative and immunomodulatory capabilities, limited ethical concerns, and low risk of tumor formation. Although several studies have shown that MSCs have the potential to mitigate the neurodegenerative pathology of PD, variabilities in preclinical and clinical trials have resulted in inconsistent therapeutic outcomes. In this review, we strive to highlight the sources of variability in studies using MSCs in PD therapy, including MSC sources, the use of autologous or allogenic MSCs, dose, delivery methods, patient factors, and measures of clinical outcome. Available evidence indicates that while the use of MSCs in PD has largely been promising, conditions need to be standardized so that studies can be effectively compared with one another and experimental designs can be improved upon, such that this body of science can continue to move forward.

npj Regenerative Medicine (2020)5:20; https://doi.org/10.1038/s41536-020-00106-y

\section{INTRODUCTION}

Parkinson's disease (PD) is the second most common neurodegenerative disease with a prevalence of $0.5-1 \%$ among people $65-69$ years of age, and rising to $1-3 \%$ among people of 80 years of age and older ${ }^{1}$. The pathological hallmarks of PD are a progressive and gradual loss of dopaminergic (DA) neurons of the nigrostriatal pathway and in the substantia nigra pars compacta ${ }^{2}$, the presence of neuronal a-synuclein inclusions called Lewy bodies, and neuro-inflammation in various brain regions ${ }^{3,4}$. The loss of DA neurons in the substantia nigra pars compacta results in typical clinical manifestation and the classification of PD as a movement disorder characterized by resting tremor, postural instability, rigidity, and bradykinesia ${ }^{5,6}$. Symptoms of PD usually appear around 55 years of age when roughly $80 \%$ of the nigrostriatal DA system is degenerated ${ }^{7,8}$. However, PD pathology extends beyond the nigrostriatal DA pathway. Lewy pathology is also found in the vagus nerve, spinal cord, sympathetic ganglia, and cardiac and myenteric plexuses ${ }^{9,10}$. This leads to a number of secondary motor and non-motor symptoms ${ }^{9,11}$ such as neuropsychiatric disorders (anxiety and depression), autonomic dysfunction (constipation), sleep abnormalities (insomnia), olfaction and visual disorders, and cognitive decline including dementia ${ }^{12,13}$.

Although the precise mechanism leading to neuronal loss in PD is still unknown, it appears to be multifactorial. The pathogenic mechanisms proposed to play a role in PD include genetic factors, excessive release of oxygen free radicals and oxidative stress, dysfunctional protein degradation, glial dysfunction, lack of trophic factors, inflammation, mitochondrial dysfunction, and accumulation of damaged mitochondria in DA neurons ${ }^{14,15}$.

The main treatments for PD patients include the administration of dopamine precursor L-3,4-dihydroxyphenylalanine (levodopa; Ldopa), dopamine receptor agonists, and inhibitors of endogenous dopamine degradation enzymes (catechol-O-methyl transferase and monoamine oxidase $B$ inhibitors), as well as surgical procedures such as deep brain stimulation (DBS) ${ }^{16}$. Pharmacological strategies can restore DA activity and improve the motor symptoms of PD, especially in the early stages of disease. However, their administration does not improve and may even exacerbate non-motor manifestations of PD, such as postural hypotension and neuropsychiatric problems ${ }^{17,18}$. In regard to the most commonly used pharmaceutical therapy, levodopa, prolonged administration results in undesirable side effects such as dyskinesia and neuropsychiatric pathologies including hallucinations and impulsive-compulsive behaviors ${ }^{19,20}$. Similar to pharmaceutical treatment, DBS is effective in controlling the motor symptoms of PD, but does not allay most of the non-motor pathological manifestations ${ }^{21}$. In order to find a cure for PD, various approaches using anti-inflammatory drugs ${ }^{22}$ and neurotrophic factors ${ }^{23}$ are being tested in preclinical models. In line with these increasing efforts to improve the efficacy of PD treatment, cell-based therapy has been raised as a promising alternative approach $^{24}$.

In this review, we describe the evolution of cell therapy in PD, highlight why mesenchymal stem/stromal cells (MSCs) are a promising option in the treatment of this disease, and stress the multitude of potential variabilities that can arise from MSC-based clinical trials in PD.

\section{EVOLUTION OF CELL THERAPY FOR TREATMENT OF PD}

Due to the selective degeneration of neurons in PD, cell-based therapies including neuronal transplantation were viewed as compelling therapeutic approaches. The proposed mechanisms of cell transplantation that allow for the mitigation of neurodegeneration and symptoms include neurite outgrowth of grafts, graft innervation, and formation of synaptic connections with the host 
Table 1. Comparison of different sources of cells for cell therapy.

\begin{tabular}{lllllll}
\hline & hESCs & iPSCs & hNSCs & iPSC-derived hNSCs & MSCs & iPSC-derived MSCs \\
\hline Ethical concerns & $!$ & $\checkmark$ & $!$ & $\checkmark$ & $\checkmark$ & $\checkmark$ \\
Genomic stability & $!$ & $!$ & $\checkmark$ & $!$ & $\checkmark$ & $\checkmark$ \\
Risk of tumor formation & $!$ & $!$ & $\checkmark$ & $!$ & $\checkmark$ & $\checkmark$ \\
Allogenic and autologous source available & $!$ & $\checkmark$ & $!$ & $\checkmark$ & $\checkmark$ \\
Risk of immunological rejection & $!$ & $\checkmark$ & $!$ & $\checkmark$ & $\checkmark$ \\
Donor-age-related issues & $\checkmark$ & $!$ & $\checkmark$ & & \\
\hline$\checkmark$ safe/advantage, ! concerns/disadvantage. & & &
\end{tabular}

tissue $^{25}$, the expression of tyrosine hydroxylase, the release of dopamine, and the release of neurotrophic and neuroprotective bioactive molecules from the transplanted cells ${ }^{26,27}$.

Over 50 years ago, the first preclinical studies involving the transplantation of rat and human-derived fetal ventral mesencephalon (hfVM) neuroblasts into rodent brains were conducted ${ }^{28}$. Subsequent studies in animal models of PD demonstrated that transplanted DA neurons obtained from the fetal midbrain were able to integrate into host tissue, release dopamine, and improve motor function. This discovery revolutionized cell-based replacement therapy as a treatment for $P D^{29}$. Based on these encouraging results, several scientific groups conducted open-label clinical trials in PD patients. However, the results were variable, with only moderate to no significant improvement ${ }^{30,31}$. These inconsistencies were largely due to highly variable trial design in addition to technical difficulties including cell type variability in tissue grafts, phenotypic instability after passaging, and poor proliferation and survival in the brain after grafting ${ }^{32,33}$. Further challenges in the use of hfVM neuroblasts in PD treatment included the host immune response to an allogenic graft, ethical concerns regarding the use of fetal tissue, and the potential for malignant transformation ${ }^{34,35}$. Due to these limitations of hfVM neuroblast-based treatment, the focus has shifted to an alternative source of cells for regenerative therapies and transplantation in PD. Recently, enormous progress has been made in the field, and several different strategies and cell sources have been tested for their potential in PD treatment including human embryonic stem cells, human induced pluripotent stem cells (hiPSCs), human neural stem cells, hiPSC-derived neural stem cells, and human MSCs. The concerns and the advantages of different stem cells sources for PD treatment are included in Table 1.

\section{THE POTENTIAL OF HMSC-BASED THERAPY FOR PD TREATMENT}

Researchers with interest in PD have focused their attention on cell-based therapies using MSCs due to their widespread availability in the body ${ }^{36}$, proliferative abilities, and immunomodulatory capabilities ${ }^{37}$. The therapeutic potential of MSCs involves several mechanisms. Human MSCs are able to secrete protective neurotrophic factors, anti-apoptotic factors, growth factors, and cytokines (vascular endothelial growth factor (VEGF), hepatocyte growth factor (HGF), insulin-like growth factor 1 (IGF-1), brainderived neurotrophic factor (BDNF), beta-nerve growth factor ( $\beta$-NGF), transforming growth factor- $\beta$ (TGF- $\beta$ ), fibroblast growth factor 2 (FGF2), and glial cell-derived neurotrophic factor (GDNF)) into a damaged, inflamed area ${ }^{38}$. In this way, MSCs could promote repair through the production of anti-inflammatory cytokines such as interleukin (IL)-10 and TGF- $\beta^{39}$ in addition to the inhibition of pro-inflammatory cytokines (tumor necrosis factor- $\alpha$, interferon gamma (IFN- $\gamma$ ), and IL-1 $\beta$ ), which have been identified to be released in the brains of PD patients ${ }^{40,41}$. MSCs also mediate hematopoiesis regulation and tissue regeneration through their paracrine signaling and multipotency, respectively ${ }^{42}$. Moreover, MSCs have the potential to be differentiated into a neural lineage, including DA neuron precursors ${ }^{43,44}$. However, it remains unclear whether undifferentiated MSCs or MSCs that have undergone neuronal differentiation are able to integrate into host neural circuits and create new synaptic connections with host neurons $^{45,46}$. Recent findings indicating that MSCs can transfer mitochondria to damaged tissue represent another intriguing mechanism that could be beneficial in PD therapy due to the proposed central role of damaged mitochondria in neurodegeneration of DA neurons ${ }^{47}$. MSC therapy has been shown to be safe with no increased risk of neoplastic transformation (Table 1$)^{48}$.

\section{THE CHALLENGES OF HMSC CELL-BASED THERAPIES FOR PD TREATMENT}

Previous clinical studies using MSCs in the treatment of PD in humans have provided promising preliminary data. In an openlabel study in 2010, autologous bone marrow (BM)-derived MSCs with a dose of $10^{6}$ cells per kilogram body weight were stereotactically administered unilaterally into the sublateral ventricular zone in seven patients with $\mathrm{PD}^{49}$. Three patients were reported to have improved PD symptoms. In 2012, the same research group started another open-label study using allogeneic BM-derived MSCs with a dose of $2 \times 10^{6}$ cells per kilogram of body weight and stereotactic administration bilaterally into the sublateral ventricular zone into eight patients with PD and eight with advanced symptoms of PD recognized as "PD plus" ${ }^{\prime 50}$. The group reported persistent improvement of symptoms in PD patients and transient improvement of symptoms in "PD plus" patients.

Currently, seven clinical trials using MSCs for PD treatment are in progress with highly variable set up (Table 2). There is a distinct lack of consistency between clinical trials, such that these studies are difficult to compare with one another in order to pinpoint what needs to be improved upon in future studies. In the following sections, we highlight the sources of variability in MSCbased PD therapy in an effort to draw attention to the need for increased standardization in this field.

\section{PD patient: selection and outcome measures}

One source of variability in the studies is the PD classification and selection of patients for the study. To date, tests allowing for the diagnosis of PD in early stages are missing. The more precise diagnosis of PD is based on the presence of substantia nigra pars compacta degeneration and Lewy pathology found during the post-mortem pathological examination. Therefore, the current diagnosis of PD is based on the symptoms that arise in the later stages of the disease, when approximately $80 \%$ of DA neurons have already been damaged.

Classification and staging of PD vary and can lead to a high degree of heterogeneity in defined PD groups. Nevertheless, classification and prediction of disease progression has significant 


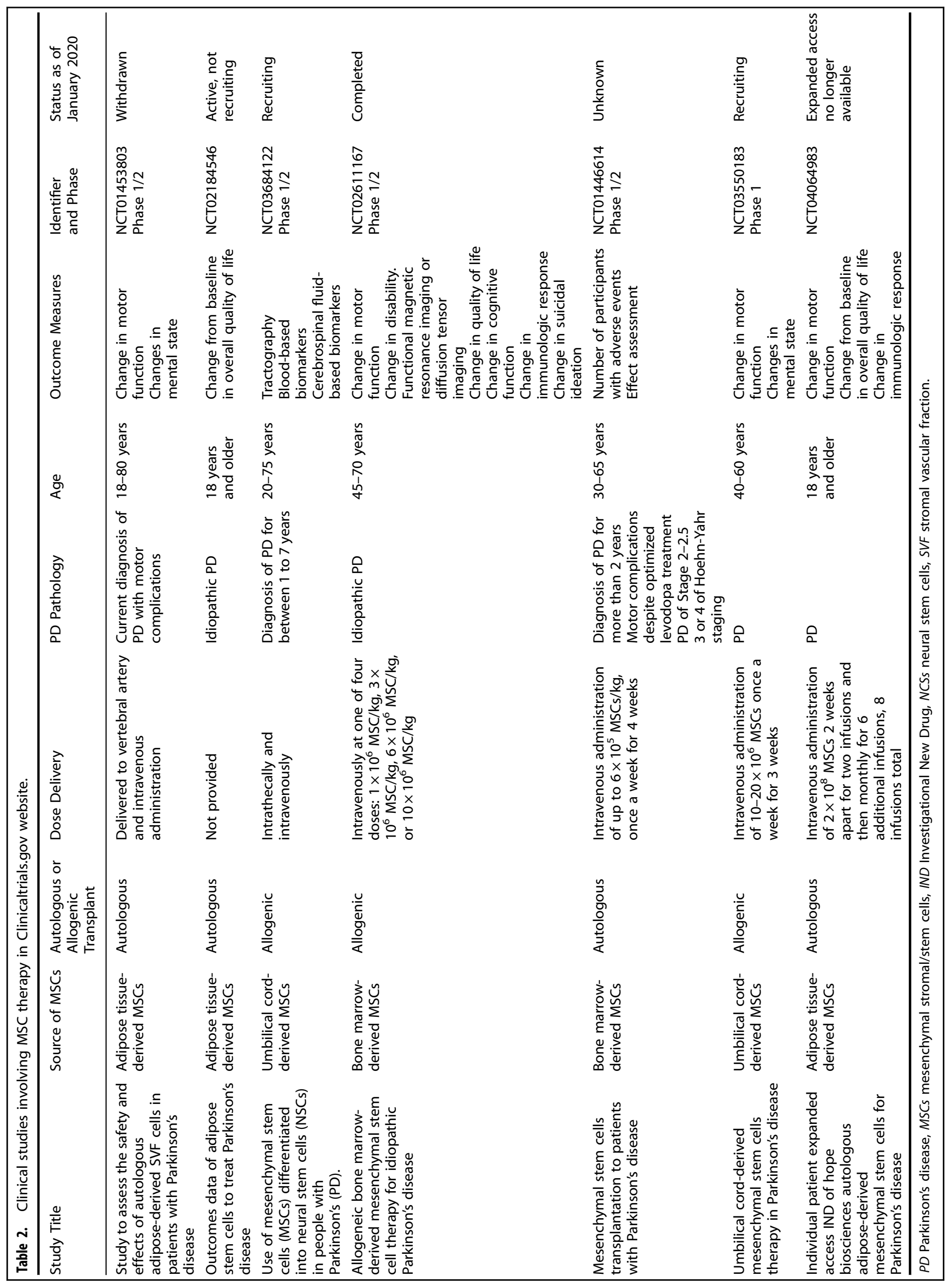


consequences for the selection of therapeutic strategies and the potential success of treatments. Currently, clinical studies focused on MSC-mediated PD treatment have mainly used age and time from diagnosis as inclusion or exclusion criteria. These studies also vary in the outcome measures for the evaluation of MSC treatment success (Table 2), although strides have recently been made to propose a global consensus of outcome measures for $\mathrm{PD}^{51}$. Moreover, these scales, which include the Hoehn and Yahr scale $^{52}$, the Unified Parkinson's Disease Rating Scale (UPDRS) ${ }^{53}$, the Movement Disorder Society-sponsored revision of the UPDRS ${ }^{54}$, NMS-Quest ${ }^{55}$, and the physician-assisted non-motor symptoms scale $^{56}$, are not only used for selection of the patient but also for measuring the clinical improvements. More objective selection criteria and improvement measures might include functional magentic resonance imaging, magnetic resonance tractography, and blood and cerebral fluid biomarkers ${ }^{57,58}$. Another important question arising from measuring the outcomes is the timing of follow-up. Thus, the use of more unified clinical groups as well as outcome measures is crucial for the successful assignment of cell therapy treatment strategies.

\section{Donor: autologous versus allogenic}

MSC-mediated treatment offers both options: autologous and allogenic transplantation. Autologous MSCs are mainly isolated from adipose tissue (AD) and BM. Variability in autologous MSC therapy between patients can stem from different aspiration techniques during harvesting, which can influence cell yield, viability, and differentiation potential ${ }^{59,60}$. Autologous MSCs are more likely to garner harvesting variabilities than allogenic MSCs, due to the need to harvest MSCs from each patient rather than having one source of expanded MSCs for experimental use. Some studies in AD-derived MSCs have found a negative correlation between donor age and cell proliferation of the $\mathrm{MSCs}^{61}$. Similarly, the yield of MSCs from BM aspirate has been found to have a negative correlation with age ${ }^{62}$. Furthermore, a study using immortalized AD-derived MSCs from PD patients have demonstrated significant mitochondrial dysfunction in the MSCs as compared to the immortalized MSCs of non-PD patients ${ }^{63}$. Additionally, several studies have proposed that PD pathology might limit the regenerative capacity of autologous MSCs due to their autophagy and decreased mitochondrial functions ${ }^{64}$. This raises concerns regarding the potential efficacy of PD patientderived MSCs. Furthermore, both pathological and clinical studies have shown that PD pathology affects not only the central nervous system (CNS) but spans through several organs, which could impact the effectiveness of autologous MSC treatment. In addition to the systemic nature of PD, the age of the patient and the systemic effect of PD medication both have important implications for autologous MSC treatment and should be taken into consideration ${ }^{65}$.

Allogenic MSCs can be isolated from umbilical cord (UC) in addition to $B M$ and $A D$. The advantages of allogenic MSC transplants include increased timely availability of cells when needed, reduced overall cost, decreased harvesting variability, and the opportunity to conduct more thorough cell quality assessments ${ }^{66}$. In contrast to autologous MSC treatment, trials involving allogenic MSCs are able to treat patients using MSCs that had undergone extensive quality-assurance measures to mitigate any batch stability or genome issues. Although cryopreservation is applicable to both autologous and allogenic MSCs, variations in freezing technique, composition of freezing media, viability and therapeutic effectiveness of MSCs after thawing represent additional sources of variation among clinical studies ${ }^{67}$. Another factor to consider in allogenic MSC transplantation is the potential for immune rejection ${ }^{66,68}$. This raised the question of whether donor human leukocyte antigen (HLA) should be matched with patients or patients should undergo immunosuppression before and/or after MSC transplantation. However, HLA matching would entail the need to have more donors, which would increase time, cost, and harvesting variables ${ }^{69}$. Additionally, immunosuppression increases the risk of cancer, infection, cardiovascular and metabolic disease, and immune dysregulation, all of which could negatively impact the lives of the patients ${ }^{70}$. Furthermore, multidose regimens have the potential to accelerate the clearance of MSCs due to the boosted allogenic memory-response ${ }^{71}$. Recent studies have suggested that the immunogenicity of MSCs have no significant adverse impact on the engraftment of MSCs in wound healing ${ }^{72}$. In general, it is believed that MSCs express a low level of HLA antigen ${ }^{73}$. However, studies have shown that major HLA class II molecules could be increased during in vitro expansion, which highlights the importance of using low-passage $\mathrm{MSCs}^{68}$. Therefore, while HLA matching may not be necessary, administering HLA-matched MSCs may prolong the survival of the MSCs in vivo. Even though allogenic MSC therapy has been shown to be well tolerated with minimal side effects, there is still concern for the risk of transmitting blood-borne pathogens, and immune rejection, which can lead to the elimination of MSCs and thus potentially lead to a reduced therapeutic effect ${ }^{66,74}$. Furthermore, an important limitation to the clinical application of both autologous and allogenic MSCs is the potential for their spontaneous differentiation into undesirable cell lineages and tissue types ${ }^{75}$.

\section{Donor: sources of MSCs}

The primary sources of MSCs are BM aspirate, $A D$, and $U C^{76,77}$. Considerations must be made regarding the selection of a source. One factor to consider for is the ease and efficiency of harvesting. The harvesting method itself can introduce experimental variability, which has the potential to significantly affect the therapeutic effectiveness of MSCs. Variability can stem from different injection sites within the same sources, or aspiration techniques, which can influence cell yield, viability, and differentiation potential ${ }^{59,60}$. Compared with $A D$ and $U C$, harvesting $B M$ is the most invasive and can cause the most pain and infection risk $^{78,79}$. BM aspirate contains only $0.001-0.01 \%$ MSCs in the overall cell population, which translates to roughly $60-600$ cells/ $\mathrm{mL}$ of aspirate $\mathrm{e}^{76}$. Considering that only a limited volume of aspirate can be withdrawn, this means that there will typically be an intensive culturing process to expand the MSCs, especially if the cells are to be used allogenically ${ }^{76,79}$. AD can be harvested from lipid waste generated from lipectomy, lipoplasty, and liposuction. AD can also be harvested from a small area under local anesthesia, making this procedure much less invasive and hazardous than BM harvesting and therefore arguably a better source for autologous use $\mathrm{e}^{79,80}$. Furthermore, an AD harvest obtains a 500 times greater yield of MSCs than an equivalent amount of $\mathrm{BM}$ aspirate ${ }^{81,82}$. Compared to $\mathrm{BM}$ and $\mathrm{AD}, \mathrm{UC}$ harvesting is the least invasive and poses no ethical challenges due to the UC tissue being derived aseptically from cesarean sections ${ }^{83,84}$. Additionally, due to the nature of UC, there are no age-related problems, which can cause issues in adult MSC isolations. Furthermore, a large amount of MSCs can be derived from one UC, which can minimize the need to extensively expand the cells for allogenic use $\mathrm{e}^{84}$. UC tissue can also be cryopreserved after harvesting, allowing for the potential to isolate MSCs as needed. However, the isolation of living MSCs from thawed, previously cryopreserved tissue is not always possible due to the differential proximity of the MSCs to the cryoprotectant ${ }^{85}$.

In addition to harvesting considerations, there are also proliferation differences between sources that need to be considered. It has been found that UC-derived MSCs proliferate faster than $\mathrm{AD}$-derived $\mathrm{MSCs}$, and $\mathrm{AD}$-derived MSCs proliferate faster than BM-derived $\mathrm{MSCs}^{83,86}$. UCderived MSCs on average have the shortest doubling time at 
$24 \mathrm{~h}^{87}$, compared to AD-derived MSCs which have a doubling time of $40 \mathrm{~h}$, and BM-derived MSCs which have a doubling time of $60 \mathrm{~h}^{79,88}$. UC-derived MSCs may be more highly proliferative due to UC-derived MSCs not being inhibited by cell-to-cell contact, which allows them to continue to proliferate even after reaching confluence $^{89}$. Additionally, differences in proliferative abilities may be impacted by the effects of senescence on MSCs. BM-derived MSCs have been found to have senescence landmarks starting at passage 7 (ref. ${ }^{90}$ ), and AD-derived MSCs starting at passage 8 (ref. ${ }^{88}$ ), which can influence the therapeutic effectiveness, number, maximum lifespan, and differentiation abilities of the cells. In contrast, UC-derived MSCs can easily be expanded over passage 16 without any landmarks of senescence and no karyotype instability or variations in morphology ${ }^{91}$.

Different sources of MSCs have also been found to have differences in their immunological characteristics, as well as have different paracrine signaling and immune modulation abilities ${ }^{92}$. By definition, MSCs should not have HLA-DR surface markers. However, studies have shown that BM and AD-derived MSCs express HLA-DR surface markers in response to IFN- $\gamma$, while UCderived MSCs do not ${ }^{93}$. Despite these findings, a study in BMderived MSCs have demonstrated that HLA-DR surface marker expression appears to be random, and their presence had little effect on the immunomodulation, multilineage differentiation, and their allogenic immune response ${ }^{94}$. Another surface marker, CD142, has been linked to thrombosis and is a concern for systemic administration of MSCs. BM-derived MSCs have been shown to have lower levels of CD142 as compared to low-passage AD-derived MSCs ${ }^{95,96}$, which could make BM-derived MSCs more suitable for intravenous MSC delivery and decrease the risk of thrombosis ${ }^{97}$. In addition to differences in surface markers, MSCs from different sources have been found to have varying paracrine functions. BM-derived MSCs have been found to have significant paracrine functions, including the secretion of angiogenic factors, growth factors, and cytokines ${ }^{98}$. It has been found that there is a lower secretion of pro-angiogenic molecules and cytokines in ADderived MSCs as compared to BM-MSCs, which suggests that ADderived MSCs might be less suited to reducing inflammation ${ }^{99}$. However, UC-derived MSCs have been found to express more angiogenic, neuroprotective, and neurogenerative factors compared to BM-derived MSCs, which make them an attractive option in PD therapy ${ }^{100}$. Furthermore, UC-derived MSCs have been found to exhibit a 20-800k relative fold change compared to BM-derived MSCs, which exhibit a 50-1000 relative fold change for indoleamine-pyrrole 2,3-dioxygenase (IDO) upregulation following IFN- $\gamma$ stimulation ${ }^{69}$. IDO suppresses T cell responses, which could help increase immune tolerance to allogenic administration of $\mathrm{MSCs}^{101}$.

Another factor that needs to be considered, especially in the treatment of PD, is the impact that the source of the MSCs has on their neuronal differentiation abilities. While BM, AD, and UCderived MSCs have all been demonstrated to express synaptophysin as evidence of the formation of a synapse ${ }^{102}, A D$-derived MSCs were exhibited to express the highest level of SAP-90 as compared to BM- and UC-derived MSCs, which indicates that ADderived MSCs may be more likely to form synaptic structures ${ }^{103}$. Likewise, BM-, AD-, and UC-derived MSCs were all capable of expressing NT-3, a neurotrophic factor, but AD-derived MSCs had the highest expression ${ }^{103}$. Furthermore, BM, AD, and UC-derived MSCs were all able to express DA neuron markers in vitro, including nurr1 and tyrosine hydroxylase, which is especially relevant in PD therapy ${ }^{103,104}$.

A potential strategy for a more objective classification of MSCs includes the analysis of their biomarkers. The minimum criteria for defining the phenotype of MSCs includes the expression of CD73, CD90, and CD105, and the lack of expression of CD45, CD34, CD14 or CD11b, CD79a or CD19, and HLA-DR ${ }^{105}$. Besides these markers, MSCs express many other surface markers and secrete various bioactive molecules including proteins, immune-modulating molecules, and microRNAs ${ }^{106}$. Multiple comprehensive transcriptomic and proteomic analyses of human MSCs have revealed different markers that may contribute to the molecular classification of subspecies of MSCs. This could lead to a more targeted approach to MSC therapy, where subspecies of MSCs are applied to specific clinical conditions that their phenotype is most suited to treating ${ }^{107}$. However, accumulating evidence suggests that marker expression of MSCs is not stable in culture conditions, which could make characterizing MSCs based on their markers a challenge ${ }^{108}$. So far, it is not clear if the classification based on novel markers can be applicable to clinical studies, but efforts remain ongoing.

Route of delivery and other variabilities

There are several routes for MSC administration in PD treatment. One option is a direct stereotactic transplantation into the affected area. In rodent PD models, direct transplantation into the striatum, substantia nigra, or subthalamic nucleus has been shown to be effective when using BM-derived MSCs ${ }^{109-114}, A D$ derived MSCs ${ }^{115-117}$, and UC-derived MSCs ${ }^{118-121}$. In some of the stereotactic transplant studies, undifferentiated MSCs were shown to differentiate into DA neurons in vivo ${ }^{109,110,118,120}$. Other studies used MSCs that were differentiated into neurons ${ }^{111,113,116,118}$, neurotrophic factors-secreting cells ${ }^{112}$, or nestin-positive stem cells ${ }^{114}$. Regardless of the PD rodent model (6-hydroxydopamine, 1-methyl-4-phenyl-1,2,3,6-tetrahydropyridine, or rotenone model), the majority of these experiments were successful, as was measured by behavioral improvement, reduced microglial activation, increased tyrosine hydroxylase immunoreactivity, neuroprotection of DA neurons, and even neurogenesis ${ }^{117,122}$. Interestingly, AD-derived MSCs did not differentiate in vivo to DA neurons in any of the studies that were reviewed ${ }^{115-117}$ (Supplementary Table 1). Although the preclinical data for direct transplantation are promising, this delivery route involves a relatively complex surgical procedure, surgery-related risks, possible post-surgical complications, inconvenient administration of repetitive doses, and high $\operatorname{costs}^{123}$.

MSCs have the ability to migrate to injury sites and promote repair, which makes them compelling candidates for systemic administration. Systemic administration of MSCs has been shown to be effective using BM-derived $\mathrm{MSCs}^{124-130}$ and $\mathrm{AD}$-derived $\mathrm{MSCs}^{126}$. In many of these studies, behavioral improvement, neuroprotective effects, and neurogeneration were observed. However, in one study, the MSCs were not able to transmigrate across the blood-brain barrier without a permeabilizing agent ${ }^{131}$, and in another study, the majority of the MSCs were found to be retained in the lungs rather than in the brain ${ }^{129}$. While some studies have shown that intravenously administered MSCs can enter the brain without the aid of a permeabilizing agent ${ }^{125,129}$, many studies did not include experiments regarding the presence of MSCs in the brain ${ }^{126,127,130}$. The less successful therapeutic outcomes observed via systemic administration of MSCs may be due to the MSCs having difficulty transmigrating across the blood-brain barrier ${ }^{132,133}$.

An alternative route for the treatment of CNS diseases is intranasal administration ${ }^{132}$. Studies in preclinical PD models using intranasal administration have demonstrated the successful delivery of MSCs to the brain with localization of MSCs in the olfactory bulb, cortex, hippocampus, striatum, cerebellum, brain stem, amygdala, hippocampus, and spinal cord even 4.5 months after injection ${ }^{134}$. Furthermore, one study has reported neuroprotective effects, anti-inflammatory effects, and improvements in neurobehavioral tests, indicating that intranasally delivered MSCs are a promising therapeutic option for $\mathrm{PD}^{135}$.

Other variabilities include questions regarding the dose, if the injections should be repeated, and, if so, how those injections 
should be timed. To date, an effective dose of MSCs for PD applications has not been optimized, and likely is different between administration routes. In the reviewed clinical trials for $\mathrm{PD}$, some studies reported doses that ranged from $6 \times 10^{5}-10 \times$ $10^{6}$ MSCs per kilogram of the patients' weight, some reported the total amount of MSCs used per patient and disregarded weight, and some studies did not report dosage at all. A meta-analysis of animal models of PD concluded that a higher dose of MSCs $(<1 \times$ $10^{6}$ versus $\geq 1 \times 10^{6} \mathrm{MSCs}$ ) did show a significant difference in effect for limb function but not in rotation behavior ${ }^{136}$, yet it is not known whether there is the same effect in non-human primates or humans. Additionally, the studies that have been conducted in primate models of PD have been largely stereotactic ${ }^{137}$, whereas the majority of clinical trials are reported to use intravenous administration, making it difficult to draw conclusions regarding dosage considerations. Standardization of dosage is necessary to limit variabilities between trials and to gain insight into what aspects needs to be improved.

\section{FUTURE DIRECTIONS AND CONCLUSIONS}

Over the decades, scientists and clinicians have put in a tremendous amount of effort into establishing stem cell therapy as an efficient and feasible treatment for neurological diseases including PD. However, systematic translational use of cell therapy is still somewhat out of reach. In order to improve the efficiency of MSC-mediated therapy, several novel strategies have been tested.

One of these approaches is MSC priming or preconditioning. Cell priming involves the exposure of cells to growth conditions that mimic the in vivo microenvironment of damaged tissue. Studies have shown that MSCs can modulate their cellular signaling in response to primed culture conditions ${ }^{138}$. This preactivation of intracellular molecular signaling before the transplantation of MSCs may improve their function, survival, and therapeutic efficacy. Several priming approaches have been tested, including priming with inflammatory cytokines or mediators, hypoxia, pharmacological drugs, chemical agents, biomaterials, and different culture conditions ${ }^{139}$. The disadvantage of this approach is the limited consensus in cell manufacturing protocols, which leads to difficulty in providing quality assurance for clinicalgrade MSCs ${ }^{140}$.

Recently, cell-free therapy has been investigated as a promising alternative approach to regenerative medicine. Several studies have established that the secretome of MSCs, which includes cytokines, growth factors, and various bioactive molecules, is what mediates their therapeutic properties. MSC-conditioned medium has been shown to have therapeutic potential in cardiovascular disease, osteoarthritis, spinal cord injury, gastric mucosal injury, and colitis $^{141,142}$. One of the components of MSC-conditioned medium is extracellular vesicles (EVs). EVs are nanovesicles that contain numerous types of proteins and RNAs, mediate communication between cells, and regulate various biological processes including immune response, angiogenesis, proliferation, and differentiation ${ }^{143}$. EVs have emerged as a key component in the MSC-mediated therapeutic response in the cardiovascular, neurological, musculoskeletal, and immune systems ${ }^{144}$. The advantage of using EVs isolated from MSC-conditioned media stems from the therapy being a cell-free approach, which requires easier administration and avoids the potentially adverse effects of cell therapy. MSC-derived EVs have been shown to provide therapeutic benefits in the treatment of various CNS disorders including stroke $^{145}$, traumatic brain injury ${ }^{146}$, Alzheimer's disease, amyotrophic lateral sclerosis, and Huntington's disease ${ }^{147}$. Moreover, it has been shown that MSC-derived EVs mediate the rescue of DA neurons in rodent PD models ${ }^{148}$. Additionally, the ability of EVs to cross the blood-brain barrier presents an attractive biological vehicle for the delivery of bioactive molecules into the brain ${ }^{149}$. However, EVs may be a part of heterogeneous populations, and their metabolomic and lipidomic profiles have not yet been well characterized. Other limitations of EV isolation and purification involve the procedure itself, which includes variability in the quality of EV preparations, the yield of EVs, and the potential for non-EV contaminants in the preparation ${ }^{150}$. Before using EVs in clinical trials, this approach still needs to be extensively evaluated for safety and efficacy.

In order to improve the generation of homogeneous, standardized, high-quality MSCs, the production of MSCs from hiPSCs has been proposed as an unlimited source of cells for therapeutic applications in regenerative medicine. Although hiPSC-derived MSCs meet the criteria for MSCs in terms of marker expression, other criteria such as the potential to differentiate to chondrogenic and adipogenic tissue are reduced compared with BMMSCs $^{151}$. The safety and efficacy of iPSC-derived MSCs are of paramount importance for successful application in the field of translational regenerative medicine. The major concerns regarding iPSC-derived MSCs include determining a suitable starter cell line ${ }^{152}$ and using a reprogramming strategy that is safe for patients. The viral vector-based strategy for reprogramming might present a potential for tumorigenic transformation ${ }^{153}$. However, recent developments in non-viral based technologies including chemicals, plasmids, and recombinant protein-based approaches might present safer strategies for the generation of iPSC-derived MSCs suitable for use in a clinical setting ${ }^{154}$.

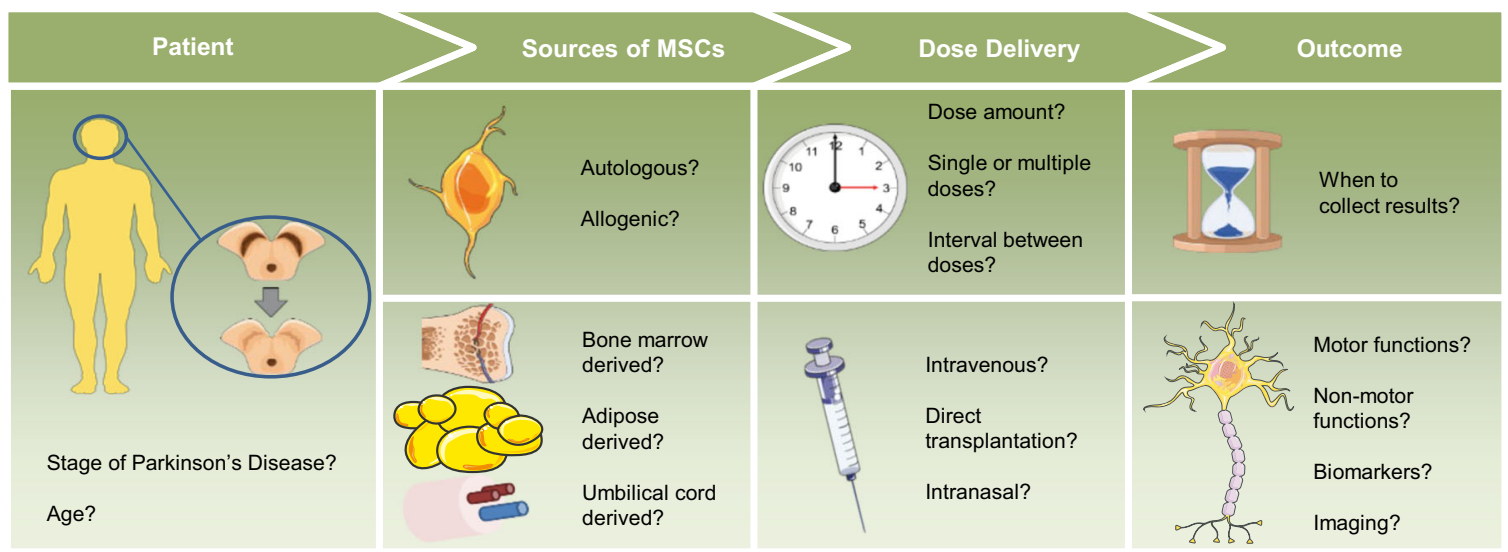

Fig. 1 Roadmap of clinical considerations regarding the use of MSCs in PD therapy. Relevant sources of variability in clinical trials for PD include patient factors, MSC sources, dose delivery, and clinical outcomes of therapy. Created using elements of Servier Medical Art by Servier, licensed under CC BY 3.0 (https://smart.servier.com/). 
Genome-edited MSCs that over-express or inhibit specific genes represent another challenging yet promising approach to improve the therapeutic properties of MSCs. Specifically for PD, several studies using engineered MSCs that expressed tyrosine hydroxylase $^{155}$, vascular endothelial growth factor ${ }^{156}$, or were transduced to produce increased glial cell-derived neurotrophic factor $^{157}$ or cerebral dopamine neurotrophic factor ${ }^{158}$ have demonstrated positive results in preclinical rodent models. However, viral transduction and genetic modification imparts added safety concerns to cell therapy, which creates additional barriers to clinical testing.

The field of cell-based therapies for PD treatment has faced several challenges. The missing or modest clinical improvement in PD patients treated with MSCs seems to have been the consequence of high variability in clinical trials. In this review, we wanted to stress that allogenic versus autologous transplantation, donor tissue source, culture conditions, PD stage, route of administration, dose, clinical evaluation criteria, and timing of evaluation are sources of variability that can lead to inconsistent results (Fig. 1). Nevertheless, MSCs harbor significant therapeutic potential for the treatment of PD. The advantages of using MSCs for PD therapy include their widespread availability and accessibility, potential for transdifferentiation into neural lineages including DA neurons, immunosuppression in the brain and inhibition of proinflammatory cytokines, migratory capacity towards damaged areas, and limited histocompatibility and ethical concerns. The experience gained in previous clinical trials should guide the future directions and emphasize the crucial need for a systematic approach to searching for optimal combinations of conditions in order to achieve reliable and effective treatment designs for PD.

Received: 20 April 2020; Accepted: 31 August 2020;

Published online: 03 November 2020

\section{REFERENCES}

1. Schneider, S. A. \& Obeso, J. A. Clinical and pathological features of Parkinson's disease. Curr. Top. Behav. Neurosci. 22, 205-220 (2015).

2. Hornykiewicz, O. Parkinson's disease: from brain homogenate to treatment. Fed. Proc. 32, 183-190 (1973).

3. Wang, Q., Liu, Y. \& Zhou, J. Neuroinflammation in Parkinson's disease and its potential as therapeutic target. Transl. Neurodegener. 4, 19 (2015).

4. More, S. V., Kumar, H., Kim, I. S., Song, S. Y. \& Choi, D. K. Cellular and molecular mediators of neuroinflammation in the pathogenesis of Parkinson's disease. Mediators Inflamm. 2013, 952375 (2013).

5. Dauer, W. \& Przedborski, S. Parkinson's disease: mechanisms and models. Neuron 39, 889-909 (2003).

6. Jankovic, J. Parkinson's disease: clinical features and diagnosis. J. Neurol. Neurosurg. Psychiatry 79, 368-376 (2008).

7. Morrish, P. K., Sawle, G. V. \& Brooks, D. J. Clinical and [18F] dopa PET findings in early Parkinson's disease. J. Neurol. Neurosurg. Psychiatry 59, 597-600 (1995).

8. Jankovic, J. \& Kapadia, A. S. Functional decline in Parkinson disease. Arch. Neurol. 58, 1611-1615 (2001).

9. Del Tredici, K. \& Braak, H. Lewy pathology and neurodegeneration in premotor Parkinson's disease. Mov. Disord. 27, 597-607 (2012).

10. Braak, H., Sastre, M., Bohl, J. R., de Vos, R. A. \& Del Tredici, K. Parkinson's disease: lesions in dorsal horn layer I, involvement of parasympathetic and sympathetic pre- and postganglionic neurons. Acta Neuropathol. 113, 421-429 (2007).

11. Bohnen, N. I. et al. Extra-nigral pathological conditions are common in Parkinson's disease with freezing of gait: an in vivo positron emission tomography study. Mov. Disord. 29, 1118-1124 (2014).

12. Gelb, D. J., Oliver, E. \& Gilman, S. Diagnostic criteria for Parkinson disease. Arch. Neurol. 56, 33-39 (1999).

13. Hughes, A. J., Daniel, S. E. \& Lees, A. J. The clinical features of Parkinson's disease in 100 histologically proven cases. Adv. Neurol. 60, 595-599 (1993).

14. Hirsch, E. C. \& Hunot, S. Neuroinflammation in Parkinson's disease: a target for neuroprotection? Lancet Neurol. 8, 382-397 (2009).

15. Truban, D., Hou, X., Caulfield, T. R., Fiesel, F. C. \& Springer, W. PINK1, Parkin, and mitochondrial quality control: what can we learn about Parkinson's disease pathobiology? J. Parkinsons Dis. 7, 13-29 (2017).
16. Savitt, J. M., Dawson, V. L. \& Dawson, T. M. Diagnosis and treatment of Parkinson disease: molecules to medicine. J. Clin. Invest. 116, 1744-1754 (2006).

17. Young, B. K., Camicioli, R. \& Ganzini, L. Neuropsychiatric adverse effects of antiparkinsonian drugs. Characteristics, evaluation and treatment. Drugs Aging 10, 367-383 (1997).

18. Kujawa, K., Leurgans, S., Raman, R., Blasucci, L. \& Goetz, C. G. Acute orthostatic hypotension when starting dopamine agonists in Parkinson's disease. Arch. Neurol. 57, 1461-1463 (2000).

19. Ernst, A. M. The role of biogenic amines in the extra-pyramidal system. Acta Physiol. Pharm. Neerl. 15, 141-154 (1969).

20. Voon, V. et al. Chronic dopaminergic stimulation in Parkinson's disease: from dyskinesias to impulse control disorders. Lancet Neurol. 8, 1140-1149 (2009).

21. Kalia, S. K., Sankar, T. \& Lozano, A. M. Deep brain stimulation for Parkinson's disease and other movement disorders. Curr. Opin. Neurol. 26, 374-380 (2013).

22. Anusha, C., Sumathi, T. \& Joseph, L. D. Protective role of apigenin on rotenone induced rat model of Parkinson's disease: suppression of neuroinflammation and oxidative stress mediated apoptosis. Chem. Biol. Interact. 269, 67-79 (2017).

23. Hegarty, S. V., Lee, D. J., O'Keeffe, G. W. \& Sullivan, A. M. Effects of intracerebral neurotrophic factor application on motor symptoms in Parkinson's disease: a systematic review and meta-analysis. Parkinsonism Relat. Disord. 38, 19-25 (2017).

24. Han, F. et al. Development of stem cell-based therapy for Parkinson's disease. Transl. Neurodegener. 4, 16 (2015).

25. Pantcheva, P., Reyes, S., Hoover, J., Kaelber, S. \& Borlongan, C. V. Treating nonmotor symptoms of Parkinson's disease with transplantation of stem cells. Expert Rev. Neurother. 15, 1231-1240 (2015).

26. Ara, J. et al. Inactivation of tyrosine hydroxylase by nitration following exposure to peroxynitrite and 1-methyl-4-phenyl-1,2,3,6-tetrahydropyridine (MPTP). Proc. Natl Acad. Sci. USA 95, 7659-7663 (1998).

27. Lindsay, R. M., Altar, C. A., Cedarbaum, J. M., Hyman, C. \& Wiegand, S. J. The therapeutic potential of neurotrophic factors in the treatment of Parkinson's disease. Exp. Neurol. 124, 103-118 (1993).

28. Björklund, A. J. Reconstruction of the nigrostriatal dopamine pathway by intracerebral nigral transplants. Brain Res. 177, 555-560 (1979).

29. Björklund, A. J. Reinnervation of the denervated striatum by substantia nigra transplants: functional consequences as revealed by pharmacological and sensorimotor testing. Brain Res. 199, 307-333 (1980).

30. Kordower, J. H. et al. Neuropathological evidence of graft survival and striatal reinnervation after the transplantation of fetal mesencephalic tissue in a patient with Parkinson's disease. N. Engl. J. Med. 332, 1118-1124 (1995).

31. Piccini, P. et al. Dopamine release from nigral transplants visualized in vivo in a Parkinson's patient. Nat. Neurosci. 2, 1137-1140 (1999).

32. Courtois, E. T. et al. In vitro and in vivo enhanced generation of human $A 9$ dopamine neurons from neural stem cells by Bcl-XL. J. Biol. Chem. 285, 9881-9897 (2010)

33. Ramos-Moreno, T., Lendinez, J. G., Pino-Barrio, M. J., Del Arco, A. \& MartinezSerrano, A. Clonal human fetal ventral mesencephalic dopaminergic neuron precursors for cell therapy research. PLoS ONE 7, e52714 (2012).

34. Ganz, J., Lev, N., Melamed, E. \& Offen, D. Cell replacement therapy for Parkinson's disease: how close are we to the clinic? Expert Rev. Neurother. 11, 1325-1339 (2011).

35. Piquet, A. L., Venkiteswaran, K., Marupudi, N. I., Berk, M. \& Subramanian, T. The immunological challenges of cell transplantation for the treatment of Parkinson's disease. Brain Res. Bull. 88, 320-331 (2012).

36. Paul, G. et al. The adult human brain harbors multipotent perivascular mesenchymal stem cells. PLoS ONE 7, e35577 (2012).

37. Paul, G. \& Anisimov, S. V. The secretome of mesenchymal stem cells: potential implications for neuroregeneration. Biochimie 95, 2246-2256 (2013).

38. Boucherie, C. \& Hermans, E. Adult stem cell therapies for neurological disorders: benefits beyond neuronal replacement? J. Neurosci. Res. 87, 1509-1521 (2009).

39. Nasef, A. et al. Identification of IL-10 and TGF- $\beta$ transcripts involved in the inhibition of T-lymphocyte proliferation during cell contact with human mesenchymal stem cells. Gene Expr. 13, 217-226 (2006).

40. Nagatsu, T., Mogi, M., Ichinose, H. \& Togari, A. Cytokines in Parkinson's disease. J. Neural Transm. Suppl 58, 143-151 (2000).

41. Tansey, M. G. \& Goldberg, M. S. Neuroinflammation in Parkinson's disease: its role in neuronal death and implications for therapeutic intervention. Neurobiol. Dis. 37, 510-518 (2010).

42. Caplan, A. I. Adult mesenchymal stem cells: when, where, and how. Stem Cells Int. 2015, 628767 (2015).

43. Joyce, N. et al. Mesenchymal stem cells for the treatment of neurodegenerative disease. Regen. Med. 5, 933-946 (2010).

44. Hayashi, T. et al. Autologous mesenchymal stem cell-derived dopaminergic neurons function in parkinsonian macaques. J. Clin. Invest. 123, 272-284 (2013). 
45. Zeng, X. et al. Integration of donor mesenchymal stem cell-derived neuron-like cells into host neural network after rat spinal cord transection. Biomaterials $\mathbf{5 3}$, 184-201 (2015).

46. Blandini, F. et al. Transplantation of undifferentiated human mesenchymal stem cells protects against 6-hydroxydopamine neurotoxicity in the rat. Cell Transpl. 19, 203-217 (2010).

47. Liu, K. et al. Mesenchymal stem cells rescue injured endothelial cells in an in vitro ischemia-reperfusion model via tunneling nanotube like structuremediated mitochondrial transfer. Microvasc. Res. 92, 10-18 (2014).

48. Centeno, C. J. et al. A multi-center analysis of adverse events among two thousand, three hundred and seventy two adult patients undergoing adult autologous stem cell therapy for orthopaedic conditions. Int. Orthop. 40, 1755-1765 (2016).

49. Venkataramana, N. K. et al. Open-labeled study of unilateral autologous bonemarrow-derived mesenchymal stem cell transplantation in Parkinson's disease. Transl. Res. 155, 62-70 (2010).

50. Venkataramana, N. K. et al. Bilateral transplantation of allogenic adult human bone marrow-derived mesenchymal stem cells into the subventricular zone of Parkinson's disease: a pilot clinical study. Stem Cells Int. 2012, 931902 (2012).

51. de Roos, P. et al. A consensus set of outcomes for Parkinson's disease from the International Consortium for Health Outcomes Measurement. J. Parkinsons Dis. 7, 533-543 (2017).

52. Hoehn, M. M. \& Yahr, M. D. Parkinsonism: onset, progression and mortality. Neurology 17, 427-442 (1967).

53. Goetz, C. G. et al. Movement Disorder Society Task Force report on the Hoehn and Yahr staging scale: status and recommendations. Mov. Disord. 19, 1020-1028 (2004).

54. Goetz, C. G. et al. Movement Disorder Society-sponsored revision of the Unified Parkinson's Disease Rating Scale (MDS-UPDRS): scale presentation and clinimetric testing results. Mov. Disord. 23, 2129-2170 (2008)

55. Chaudhuri, K. R. et al. International multicenter pilot study of the first comprehensive self-completed nonmotor symptoms questionnaire for Parkinson's disease: the NMSQuest study. Mov. Disord. 21, 916-923 (2006).

56. Chaudhuri, K. R. et al. The metric properties of a novel non-motor symptoms scale for Parkinson's disease: results from an international pilot study. Mov. Disord. 22, 1901-1911 (2007).

57. Prange, S., Metereau, E. \& Thobois, S. Structural imaging in Parkinson's disease: new developments. Curr. Neurol. Neurosci. Rep. 19, 50 (2019).

58. Jimenez-Jimenez, F. J., Alonso-Navarro, H., Garcia-Martin, E. \& Agundez, J. A. Cerebrospinal fluid biochemical studies in patients with Parkinson's disease: toward a potential search for biomarkers for this disease. Front. Cell Neurosci. 8, 369 (2014)

59. Wilson, A., Butler, P. E. \& Seifalian, A. M. Adipose-derived stem cells for clinical applications: a review. Cell Prolif. 44, 86-98 (2011).

60. Bowen, J. E. Technical issues in harvesting and concentrating stem cells (bone marrow and adipose). PMR 7, S8-S18 (2015).

61. Maredziak, M., Marycz, K., Tomaszewski, K. A., Kornicka, K. \& Henry, B. M. The influence of aging on the regenerative potential of human adipose derived mesenchymal stem cells. Stem Cells Int. 2016, 2152435 (2016).

62. Stolzing, A., Jones, E., McGonagle, D. \& Scutt, A. Age-related changes in human bone marrow-derived mesenchymal stem cells: consequences for cell therapies. Mech. Ageing Dev. 129, 163-173 (2008).

63. Moon, H. E. et al. Mitochondrial dysfunction of immortalized human adipose tissue-derived mesenchymal stromal cells from patients with Parkinson's disease. Exp. Neurobiol. 22, 283-300 (2013).

64. Angelova, P. R. et al. Mitochondrial dysfunction in Parkinsonian mesenchymal stem cells impairs differentiation. Redox Biol. 14, 474-484 (2018).

65. Yasuhara, T., Kameda, M., Sasaki, T., Tajiri, N. \& Date, I. Cell therapy for Parkinson's disease. Cell Transpl. 26, 1551-1559 (2017).

66. Griffin, M. D., Ritter, T. \& Mahon, B. P. Immunological aspects of allogeneic mesenchymal stem cell therapies. Hum. Gene Ther. 21, 1641-1655 (2010).

67. Marquez-Curtis, L. A., Janowska-Wieczorek, A., McGann, L. E. \& Elliott, J. A. Mesenchymal stromal cells derived from various tissues: biological, clinical and cryopreservation aspects. Cryobiology 71, 181-197 (2015).

68. Berglund, A. K., Fortier, L. A., Antczak, D. F. \& Schnabel, L. V. Immunoprivileged no more: measuring the immunogenicity of allogeneic adult mesenchymal stem cells. Stem Cell Res. Ther. 8, 288 (2017).

69. Mennan, C., Garcia, J., Roberts, S., Hulme, C. \& Wright, K. A comprehensive characterisation of large-scale expanded human bone marrow and umbilical cord mesenchymal stem cells. Stem Cell Res. Ther. 10, 99 (2019).

70. Riminton, D. S., Hartung, H. P. \& Reddel, S. W. Managing the risks of immunosuppression. Curr. Opin. Neurol. 24, 217-223 (2011).

71. Zhang, J. et al. The challenges and promises of allogeneic mesenchymal stem cells for use as a cell-based therapy. Stem Cell Res. Ther. 6, 234 (2015).
72. Chen, L., Tredget, E. E., Liu, C. \& Wu, Y. Analysis of allogenicity of mesenchymal stem cells in engraftment and wound healing in mice. PLOS ONE 4, e7119 (2009).

73. Uccelli, A., Moretta, L. \& Pistoia, V. Mesenchymal stem cells in health and disease. Nat. Rev. Immunol. 8, 726-736 (2008).

74. Uhlin, M. et al. Risk factors for Epstein-Barr virus-related post-transplant lymphoproliferative disease after allogeneic hematopoietic stem cell transplantation. Haematologica 99, 346-352 (2014).

75. Chamberlain, G., Fox, J., Ashton, B. \& Middleton, J. Concise review: mesenchymal stem cells: their phenotype, differentiation capacity, immunological features, and potential for homing. Stem Cells 25, 2739-2749 (2007).

76. Pittenger, M. F. et al. Multilineage potential of adult human mesenchymal stem cells. Science 284, 143-147 (1999).

77. Wexler, S. A. et al. Adult bone marrow is a rich source of human mesenchymal 'stem' cells but umbilical cord and mobilized adult blood are not. Br. J. Haematol. 121, 368-374 (2003).

78. Juneja, S. C., Viswanathan, S., Ganguly, M. \& Veillette, C. A simplified method for the aspiration of bone marrow from patients undergoing hip and knee joint replacement for isolating mesenchymal stem cells and in vitro chondrogenesis. Bone Marrow Res. 2016, 3152065 (2016).

79. Mastrolia, I. et al. Challenges in clinical development of mesenchymal stromal/ stem cells: concise review. Stem Cells Transl. Med. 8, 1135-1148 (2019).

80. Kolaparthy, L. K., Sanivarapu, S., Moogla, S. \& Kutcham, R. S. Adipose tissueadequate, accessible regenerative material. Int. J. Stem Cells 8, 121-127 (2015).

81. Strioga, M., Viswanathan, S., Darinskas, A., Slaby, O. \& Michalek, J. Same or not the same? Comparison of adipose tissue-derived versus bone marrow-derived mesenchymal stem and stromal cells. Stem Cells Dev. 21, 2724-2752 (2012).

82. Sabol, R. A. et al. Therapeutic potential of adipose stem cells. Adv. Exp. Med. Biol. https://doi.org/10.1007/5584_2018_248 (2018).

83. Mahmood, R., Shaukat, M. \& Choudhery, M. S. Biological properties of mesenchymal stem cells derived from adipose tissue, umbilical cord tissue and bone marrow. AIMS Cell Tissue Eng. 2, 78-90 (2018).

84. Jin, H. J. et al. Comparative analysis of human mesenchymal stem cells from bone marrow, adipose tissue, and umbilical cord blood as sources of cell therapy. Int. J. Mol. Sci. 14, 17986-18001 (2013).

85. Fong, C. Y., Subramanian, A., Biswas, A. \& Bongso, A. Freezing of fresh Wharton's jelly from human umbilical cords yields high post-thaw mesenchymal stem cell numbers for cell-based therapies. J. Cell Biochem. 117, 815-827 (2016).

86. Li, C. Y. et al. Comparative analysis of human mesenchymal stem cells from bone marrow and adipose tissue under xeno-free conditions for cell therapy. Stem Cell Res. Ther. 6, 55 (2015).

87. Pirjali, T. et al. Isolation and Characterization of human mesenchymal stem cells derived from human umbilical cord Wharton's jelly and amniotic membrane. Int J. Organ Transpl. Med. 4, 111-116 (2013).

88. Peng, L. et al. Comparative analysis of mesenchymal stem cells from bone marrow, cartilage, and adipose tissue. Stem Cells Dev. 17, 761-773 (2008).

89. Choudhery, M. S., Badowski, M., Muise, A. \& Harris, D. T. Comparison of human mesenchymal stem cells derived from adipose and cord tissue. Cytotherapy 15, 330-343 (2013).

90. Stenderup, K., Justesen, J., Clausen, C. \& Kassem, M. Aging is associated with decreased maximal life span and accelerated senescence of bone marrow stromal cells. Bone 33, 919-926 (2003).

91. Zhang, X. et al. Isolation and characterization of mesenchymal stem cells from human umbilical cord blood: reevaluation of critical factors for successful isolation and high ability to proliferate and differentiate to chondrocytes as compared to mesenchymal stem cells from bone marrow and adipose tissue. J. Cell Biochem. 112, 1206-1218 (2011).

92. Russell, A. L., Lefavor, R., Durand, N., Glover, L. \& Zubair, A. C. Modifiers of mesenchymal stem cell quantity and quality. Transfusion 58, 1434-1440 (2018).

93. Kim, J. H., Jo, C. H., Kim, H. R. \& Hwang, Y. I. Comparison of immunological characteristics of mesenchymal stem cells from the periodontal ligament, umbilical cord, and adipose tissue. Stem Cells Int. 2018, 8429042 (2018).

94. Grau-Vorster, M., Laitinen, A., Nystedt, J. \& Vives, J. HLA-DR expression in clinicalgrade bone marrow-derived multipotent mesenchymal stromal cells: a two-site study. Stem Cell Res. Ther. 10, 164 (2019).

95. Christy, B. A. et al. Procoagulant activity of human mesenchymal stem cells. J. Trauma Acute Care Surg. 83, S164-S169 (2017).

96. Le Blanc, K. \& Davies, L. C. MSCs-cells with many sides. Cytotherapy 20, 273-278 (2018).

97. Moll, G. et al. Are therapeutic human mesenchymal stromal cells compatible with human blood? Stem Cells 30, 1565-1574 (2012).

98. Kwon, H. M. et al. Multiple paracrine factors secreted by mesenchymal stem cells contribute to angiogenesis. Vasc. Pharm. 63, 19-28 (2014).

99. Elman, J. S., Li, M., Wang, F., Gimble, J. M. \& Parekkadan, B. A comparison of adipose and bone marrow-derived mesenchymal stromal cell secreted factors in the treatment of systemic inflammation. J. Inflamm. (Lond.) 11, 1 (2014). 
100. Hsieh, J. Y. et al. Mesenchymal stem cells from human umbilical cord express preferentially secreted factors related to neuroprotection, neurogenesis, and angiogenesis. PLOS ONE 8, e72604 (2013).

101. Le Rond, S., Gonzalez, A., Gonzalez, A. S., Carosella, E. D. \& Rouas-Freiss, N. Indoleamine 2,3 dioxygenase and human leucocyte antigen-G inhibit the T-cell alloproliferative response through two independent pathways. Immunology 116, 297-307 (2005)

102. Hayase, M. et al. Committed neural progenitor cells derived from genetically modified bone marrow stromal cells ameliorate deficits in a rat model of stroke. J. Cereb. Blood Flow. Metab. 29, 1409-1420 (2009).

103. Urrutia, D. N. et al. Comparative study of the neural differentiation capacity of mesenchymal stromal cells from different tissue sources: an approach for their use in neural regeneration therapies. PLOS ONE 14, e0213032 (2019).

104. Tatard, V. M. et al. Neurotrophin-directed differentiation of human adult marrow stromal cells to dopaminergic-like neurons. Bone 40, 360-373 (2007)

105. Dominici, M. et al. Minimal criteria for defining multipotent mesenchymal stromal cells. The International Society for Cellular Therapy position statement. Cytotherapy 8, 315-317 (2006).

106. Kozlowska, U. et al. Similarities and differences between mesenchymal stem/ progenitor cells derived from various human tissues. World J. Stem Cells 11 347-374 (2019)

107. Billing, A. M. et al. Comprehensive transcriptomic and proteomic characterization of human mesenchymal stem cells reveals source specific cellular markers. Sci. Rep. 6, 21507 (2016).

108. Lv, F. J., Tuan, R. S., Cheung, K. M. \& Leung, V. Y. Concise review: the surface markers and identity of human mesenchymal stem cells. Stem Cells 32 1408-1419 (2014)

109. Shetty, P. et al. Clinical grade mesenchymal stem cells transdifferentiated under xenofree conditions alleviates motor deficiencies in a rat model of Parkinson's disease. Cell Biol. Int. 33, 830-838 (2009).

110. Li, Y. et al. Intracerebral transplantation of bone marrow stromal cells in a 1methyl-4-phenyl-1,2,3,6-tetrahydropyridine mouse model of Parkinson's disease. Neurosci. Lett. 316, 67-70 (2001).

111. Bouchez, G. et al. Partial recovery of dopaminergic pathway after graft of adult mesenchymal stem cells in a rat model of Parkinson's disease. Neurochem. Int. 52, 1332-1342 (2008)

112. Sadan, O. et al. Protective effects of neurotrophic factor-secreting cells in a 6OHDA rat model of Parkinson disease. Stem Cells Dev. 18, 1179-1190 (2009).

113. Levy, Y. S. et al. Regenerative effect of neural-induced human mesenchymal stromal cells in rat models of Parkinson's disease. Cytotherapy 10, 340-352 (2008).

114. Ye, M. et al. Therapeutic effects of differentiated bone marrow stromal cell transplantation on rat models of Parkinson's disease. Parkinsonism Relat. Disord. 13, 44-49 (2007).

115. Schwerk, A. et al. Adipose-derived human mesenchymal stem cells induce longterm neurogenic and anti-inflammatory effects and improve cognitive but not motor performance in a rat model of Parkinson's disease. Regen. Med. 10, 431-446 (2015)

116. McCoy, M. K. et al. Autologous transplants of Adipose-Derived Adult Stromal (ADAS) cells afford dopaminergic neuroprotection in a model of Parkinson's disease. Exp. Neurol. 210, 14-29 (2008).

117. Schwerk, A. et al. Human adipose-derived mesenchymal stromal cells increase endogenous neurogenesis in the rat subventricular zone acutely after 6hydroxydopamine lesioning. Cytotherapy 17, 199-214 (2015).

118. Shetty, P., Thakur, A. M. \& Viswanathan, C. Dopaminergic cells, derived from a high efficiency differentiation protocol from umbilical cord derived mesenchymal stem cells, alleviate symptoms in a Parkinson's disease rodent model. Cell Biol. Int. 37, 167-180 (2013).

119. Weiss, M. L. et al. Human umbilical cord matrix stem cells: preliminary characterization and effect of transplantation in a rodent model of Parkinson's disease. Stem Cells 24, 781-792 (2006).

120. Xiong, $N$. et al. Long-term efficacy and safety of human umbilical cord mesenchymal stromal cells in rotenone-induced hemiparkinsonian rats. Biol. Blood Marrow Transpl. 16, 1519-1529 (2010).

121. Mathieu, P., Roca, V., Gamba, C., Del Pozo, A. \& Pitossi, F. Neuroprotective effects of human umbilical cord mesenchymal stromal cells in an immunocompetent animal model of Parkinson's disease. J. Neuroimmunol. 246, 43-50 (2012).

122. Cova, L. et al. Multiple neurogenic and neurorescue effects of human mesenchymal stem cell after transplantation in an experimental model of Parkinson's disease. Brain Res. 1311, 12-27 (2010).

123. Smith, Y., Wichmann, T., Factor, S. A. \& DeLong, M. R. Parkinson's disease therapeutics: new developments and challenges since the introduction of levodopa. Neuropsychopharmacology 37, 213-246 (2012).

124. Park, H. J., Lee, P. H., Bang, O. Y., Lee, G. \& Ahn, Y. H. Mesenchymal stem cells therapy exerts neuroprotection in a progressive animal model of Parkinson's disease. J. Neurochem. 107, 141-151 (2008).
125. Capitelli, C. S. et al. Opposite effects of bone marrow-derived cells transplantation in MPTP-rat model of Parkinson's disease: a comparison study of mononuclear and mesenchymal stem cells. Int. J. Med. Sci. 11, 1049-1064 (2014).

126. Choi, H. S. et al. Therapeutic potentials of human adipose-derived stem cells on the mouse model of Parkinson's disease. Neurobiol. Aging 36, 2885-2892 (2015).

127. Kim, Y. J. et al. Neuroprotective effects of human mesenchymal stem cells on dopaminergic neurons through anti-inflammatory action. Glia 57, 13-23 (2009).

128. Park, H. J., Shin, J. Y., Lee, B. R., Kim, H. O. \& Lee, P. H. Mesenchymal stem cells augment neurogenesis in the subventricular zone and enhance differentiation of neural precursor cells into dopaminergic neurons in the substantia nigra of a parkinsonian model. Cell Transpl. 21, 1629-1640 (2012).

129. Wang, F. et al. Intravenous administration of mesenchymal stem cells exerts therapeutic effects on parkinsonian model of rats: focusing on neuroprotective effects of stromal cell-derived factor-1alpha. BMC Neurosci. 11, 52 (2010).

130. Park, B. N., Kim, J. H., Lee, K., Park, S. H. \& An, Y. S. Improved dopamine transporter binding activity after bone marrow mesenchymal stem cell transplantation in a rat model of Parkinson's disease: small animal positron emission tomography study with F-18 FP-CIT. Eur. Radiol. 25, 1487-1496 (2015).

131. Cerri, S. et al. Intracarotid infusion of mesenchymal stem cells in an animal model of Parkinson's disease, focusing on cell distribution and neuroprotective and behavioral effects. Stem Cells Transl. Med. 4, 1073-1085 (2015).

132. Hanson, L. R. \& Frey, W. H. II Intranasal delivery bypasses the blood-brain barrier to target therapeutic agents to the central nervous system and treat neurodegenerative disease. BMC Neurosci. 9, S5 (2008).

133. Ruigrok, M. J. \& de Lange, E. C. Emerging insights for translational pharmacokinetic and pharmacokinetic-pharmacodynamic studies: towards prediction of nose-to-brain transport in humans. AAPS J. 17, 493-505 (2015).

134. Danielyan, L. et al. Intranasal delivery of bone marrow-derived mesenchymal stem cells, macrophages, and microglia to the brain in mouse models of Alzheimer's and Parkinson's disease. Cell Transpl. 23, S123-S139 (2014).

135. Salama, M. et al. Effect of intranasal stem cell administration on the nigrostriatal system in a mouse model of Parkinson's disease. Exp. Ther. Med. 13, 976-982 (2017).

136. Riecke, J. et al. A meta-analysis of mesenchymal stem cells in animal models of Parkinson's disease. Stem Cells Dev. 24, 2082-2090 (2015).

137. Gugliandolo, A., Bramanti, P. \& Mazzon, E. Mesenchymal stem cell therapy in Parkinson's disease animal models. Curr. Res. Transl. Med. 65, 51-60 (2017).

138. Durand, N., Russell, A. \& Zubair, A. C. Effect of comedications and endotoxins on mesenchymal stem cell secretomes, migratory and immunomodulatory capacity. J. Clin. Med. 8, https://doi.org/10.3390/jcm8040497 (2019).

139. Le Blanc, K. \& Mougiakakos, D. Multipotent mesenchymal stromal cells and the innate immune system. Nat. Rev. Immunol. 12, 383-396 (2012).

140. Yin, J. Q., Zhu, J. \& Ankrum, J. A. Manufacturing of primed mesenchymal stromal cells for therapy. Nat. Biomed. Eng. 3, 90-104 (2019).

141. Wang, Y., Chen, X., Cao, W. \& Shi, Y. Plasticity of mesenchymal stem cells in immunomodulation: pathological and therapeutic implications. Nat. Immunol. 15, 1009-1016 (2014).

142. Huang, P. et al. Mechanism of mesenchymal stem cell-induced neuron recovery and anti-inflammation. Cytotherapy 16, 1336-1344 (2014).

143. Kalluri, R. \& LeBleu, V. S. The biology, function, and biomedical applications of exosomes. Science 367, https://doi.org/10.1126/science.aau6977 (2020)

144. Reiner, A. T. et al. Concise review: developing best-practice models for the therapeutic use of extracellular vesicles. Stem Cells Transl. Med. 6, 1730-1739 (2017).

145. Xin, $H$. et al. Systemic administration of exosomes released from mesenchymal stromal cells promote functional recovery and neurovascular plasticity after stroke in rats. J. Cereb. Blood Flow Metab. 33, 1711-1715 (2013).

146. Zhang, Y. et al. Effect of exosomes derived from multipluripotent mesenchymal stromal cells on functional recovery and neurovascular plasticity in rats after traumatic brain injury. J. Neurosurg. 122, 856-867 (2015).

147. Yuan, Q., Li, X., Zhang, S., Wang, H. \& Wang, Y. Extracellular vesicles in neurodegenerative diseases: insights and new perspectives. Genes Dis. https://doi.org/ 10.1016/j.gendis.2019.12.001 (2019).

148. Jarmalaviciute, A., Tunaitis, V., Pivoraite, U., Venalis, A. \& Pivoriunas, A. Exosomes from dental pulp stem cells rescue human dopaminergic neurons from 6hydroxy-dopamine-induced apoptosis. Cytotherapy 17, 932-939 (2015).

149. Alvarez-Erviti, L. et al. Delivery of siRNA to the mouse brain by systemic injection of targeted exosomes. Nat. Biotechnol. 29, 341-345 (2011).

150. Wiest, E. F. \& Zubair, A. C. Challenges of manufacturing mesenchymal stromal cell-derived extracellular vesicles in regenerative medicine. Cytotherapy https:// doi.org/10.1016/j.jcyt.2020.04.040 (2020).

151. Xu, M., Shaw, G., Murphy, M. \& Barry, F. Induced pluripotent stem cell-derived mesenchymal stromal cells are functionally and genetically different from bone marrow-derived mesenchymal stromal cells. Stem Cells 37, 754-765 (2019). 
152. Jung, Y., Bauer, G. \& Nolta, J. A. Concise review: Induced pluripotent stem cellderived mesenchymal stem cells: progress toward safe clinical products. Stem Cells 30, 42-47 (2012).

153. Nakagawa, M., Takizawa, N., Narita, M., Ichisaka, T. \& Yamanaka, S. Promotion of direct reprogramming by transformation-deficient Myc. Proc. Natl Acad. Sci. USA 107, 14152-14157 (2010).

154. Sabapathy, V. \& Kumar, S. hiPSC-derived iMSCs: NextGen MSCs as an advanced therapeutically active cell resource for regenerative medicine. J. Cell Mol. Med. 20, 1571-1588 (2016).

155. Lu, L. et al. Therapeutic benefit of TH-engineered mesenchymal stem cells for Parkinson's disease. Brain Res. Brain Res. Protoc. 15, 46-51 (2005).

156. Xiong, N. et al. VEGF-expressing human umbilical cord mesenchymal stem cells, an improved therapy strategy for Parkinson's disease. Gene Ther. 18, 394-402 (2011).

157. Glavaski-Joksimovic, A. et al. Glial cell line-derived neurotrophic factor-secreting genetically modified human bone marrow-derived mesenchymal stem cells promote recovery in a rat model of Parkinson's disease. J. Neurosci. Res. 88, 2669-2681 (2010).

158. Mei, J. \& Niu, C. Effects of engineered conserved dopamine neurotrophic factorexpressing bone marrow stromal cells on dopaminergic neurons following 6OHDA administrations. Mol. Med. Rep. 11, 1207-1213 (2015).

\section{ACKNOWLEDGEMENTS}

This work was supported by the Center for Regenerative Medicine, Mayo Clinic.

\section{AUTHOR CONTRIBUTIONS}

D.F. and J.A.K. wrote and revised the paper. A.C.Z. designed, revised, and supervised the writing of this paper.

\section{COMPETING INTERESTS}

The authors declare no competing interests.

\section{ADDITIONAL INFORMATION}

Supplementary information is available for this paper at https://doi.org/10.1038/ s41536-020-00106-y.

Correspondence and requests for materials should be addressed to A.C.Z.

Reprints and permission information is available at http://www.nature.com/ reprints

Publisher's note Springer Nature remains neutral with regard to jurisdictional claims in published maps and institutional affiliations.

(i) Open Access This article is licensed under a Creative Commons adaptation, distribution and reproduction in any medium or format, as long as you give appropriate credit to the original author(s) and the source, provide a link to the Creative Commons license, and indicate if changes were made. The images or other third party material in this article are included in the article's Creative Commons license, unless indicated otherwise in a credit line to the material. If material is not included in the article's Creative Commons license and your intended use is not permitted by statutory regulation or exceeds the permitted use, you will need to obtain permission directly from the copyright holder. To view a copy of this license, visit http://creativecommons. org/licenses/by/4.0/.

(c) The Author(s) 2020 